\title{
NOTAS SOBRE LA DEMOGRAFIA Y LA ECONOMIA DE CATALUÑA
}

\author{
$312: 33$ (46 Cataluña)
}

por

\section{Ignacio Ballester Ros}

SUMARIO: I. INTRODUCCION.-II. LA DEMOGRAFIA: PRINCIPALES

CARACTERISTICAS DE SU POBLACION: 1. LA EVOLUCIÓN DE LA POBLACIÓN Y LOS COMPONENTES DE SU CRECIMIENTO DEMOGRÁFICO. 2. LAS TASAS DE NATALIDAD, MORTALIDAD Y NUPCIALIDAD. 3. LA DENSIDAd DE POBLACIÓN, LA LOCALIZACIÓN DE LA POBLACIÓN SEGÚN EL TAMAÑO DEMOGŔAFICO DE LOS MUNICIPIOS Y LAS TASAS DE URBANIZACIÓN. 4. La NATURALEZA DE LA POBLACIÓN Y LAS MIGRACIONES INTERIORES. 5. LA ESTRUCTURA DE LA POBLACIÓN POR SEXO Y EDAD Y LA COMPOSICIÓN DE LA POBLACION ECONÓMICAMENTE ACTIVA.-III. LA ACTIVIDAD ECONOMICA: AGRARIA, INDUSTRIAL Y DE SERVICIOS.-IV. LA RENTA Y EL CONSUMO.

\section{INTRODUCCION}

En el número 180 de esta Revista se publicó un trabajo acerca del medio físico, la demografía y la renta en Galicia, que nos permitió conocer la estructura y evolución de su población y la composición y evolución de la renta.

Tenemos hoy la posibilidad de presentar una información más amplia referida a Cataluña. Con ello podremos tener la visión comparativa de dos Regiones geográficamente alejadas en el área terri- 
torial de la Península y que ofrecen un dispar grado de desarrollo económico y social.

La información de referencia ha sido publicada por el Consorcio de Información y Documentación de Cataluña en una monografía especial, bajo el título "Cataluña en cifras", y comprende abundantes datos sobre los aspectos demográficos y económicos de la Región catalana. Se trata, como se indica en las notas metodológicas que se presentan a manera de introducción, de «una obra que abarca muchos temas sin pretender la exhaustividad de perspectiva en cada uno de ellos, pero con la suficiente cobertura para lograr una visión amplia de la realidad que se describe» dentro de la limitación que la disponibilidad de información estadística plantea.

Comprende quince capítulos, la mayor parte de cuyos datos proceden del Instituto Nacional de Estadística y de los distintos Ministerios, sobre los que se ha realizado una selección temática, con búsqueda retrospectiva de fuentes y con cálculos simples de agregación, tasas e índices, definición de términos y tabulación. Esta última se contempla bajo una doble perspectiva y abarca, como mínimo, una tabla sobre estructura y otra con una corta serie temporal de evolución reciente. Las tablas, de doble entrada, presentan los datos referidos sistemáticamente a siete unidades territoriales: cada una de las Provincias de Barcelona, Gerona, Lérida y Tarragona, por este orden; Cataluña en conjunto, España y el resto de España. La profusión de cifras absolutas y de las relativas correspondientes facilita mucho su análisis.

Trataremos de ofrecer y comentar los datos de mayor interés, entresacados de esta riqueza informativa, que no puede abordarse plenamente en un trabajo, de forzada limitación de espacio, como el presente.

\section{LA DEMOGRAFIA: PRINCIPALES CARACTERISTICAS DE SU POBLACION}

Según el Censo de Población de España de 1970, la población de Cataluña se elevaba a 5.122.567 habitantes, que equivale al 15,1 por 100 de la población española, y se distribuye en sus cuatro Provincias en esta forma: 


\begin{tabular}{|c|c|c|}
\hline & Habitantes & Porcentajes \\
\hline 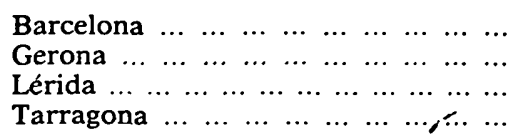 & $\begin{array}{r}3.939 .194 \\
414.397 \\
347.015 \\
431.961\end{array}$ & $\begin{array}{r}76,7 \\
8,1 \\
6,8 \\
8,4\end{array}$ \\
\hline
\end{tabular}

De ello se deduce que, si bien Cataluña dispone de la sexta parte de la población española, se advierte, de otro lado, una fuerte concentración demográfica en la Provincia de Barcelona, en la que residen prácticamente tres de cada cuatro habitantes de la Región. Queda, de esta forma, patente el reducido peso demográfico de las tres Provincias restantes.

Trataremos de destacar los aspectos básicos de su estructura y evolución demográfica.

1. LA EVOLUCión DE LA POBLACIÓN Y LOS COMPONENTES DE SU CRECIMIENTO DEMOGRAFICO

La evolución de la población de Cataluña se refleja en las siguientes cifras:

\begin{tabular}{|c|c|c|}
\hline \multirow[b]{4}{*}{ 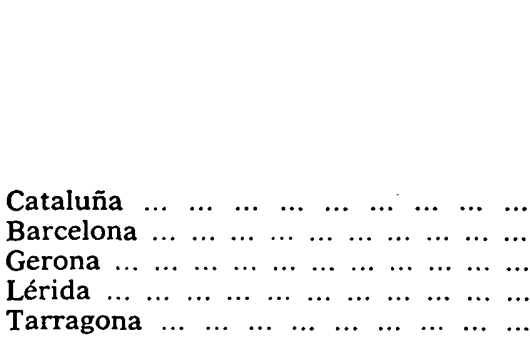 } & \multicolumn{2}{|c|}{ INCREMENTOS } \\
\hline & $1951-1960$ & $1961-1970$ \\
\hline & \multicolumn{2}{|c|}{ Cifras absolutas } \\
\hline & $\begin{array}{r}685.466 \\
645.847 \\
24.048 \\
9.703 \\
5.868\end{array}$ & $\begin{array}{r}1.196 .788 \\
1.051 .228 \\
63.028 \\
13.250 \\
69.282\end{array}$ \\
\hline & \multicolumn{2}{|c|}{ Porcentajes } \\
\hline 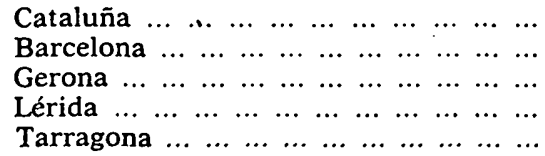 & $\begin{array}{r}21,2 \\
28,9 \\
7,3 \\
3,0 \\
1,6\end{array}$ & $\begin{array}{r}30,5 \\
36,5 \\
17,9 \\
4,0 \\
19,1\end{array}$ \\
\hline
\end{tabular}

El incremento medio nacional, cifrado en el 8,8 por 100 para el primer decenio considerado, fue ampliamente superado por $\mathrm{Ca}$ taluña en conjunto y por la Provincia de Barcelona; las otras tres 
Provincias quedaron con un ritmo muy bajo de crecimiento. En el decenio siguiente, el incremento medio nacional fue del 11,2 y fue también ampliamente superado, con un ritmo más intenso de crecimiento, por Cataluña como conjunto; por Barcelona, que vio incrementada su población en una tercera parte, y también por las Provincias de Tarragona y Gerona, que superaron acusadamente el crecimiento nacional. Tan sólo la Provincia interior de Lérida no se sumó a este fenómeno de incremento general de la población.

El crecimiento demográfico tiene dos componentes: el crecimiento vegetativo y el crecimiento migratorio. Las cifras expresivas de tales incrementos y sus valores relativos son:

\begin{tabular}{|c|c|c|}
\hline & $1951-1960$ & $1961-1970$ \\
\hline . & \multicolumn{2}{|c|}{ CRECIMIENTO VEGETATIVO } \\
\hline & \multicolumn{2}{|c|}{ Cifras absolutas } \\
\hline 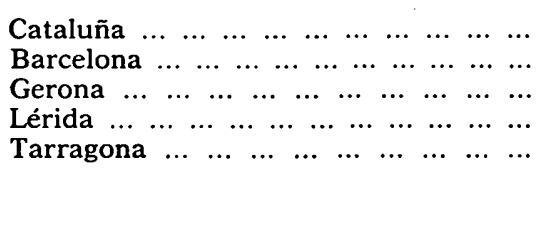 & $\begin{array}{r}215.660 \\
166.234 \\
13.678 \\
21.492 \\
14.256 \\
\\
\text { Porcen }\end{array}$ & $\begin{array}{r}476.308 \\
401.618 \\
23.951 \\
25.126 \\
25.613 \\
\\
\text { tajes }\end{array}$ \\
\hline 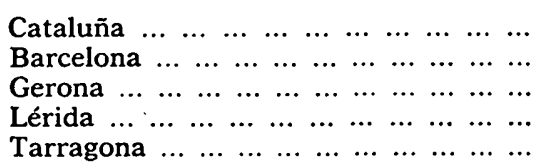 & $\begin{array}{l}6,7 \\
7,4 \\
4,2 \\
6,6 \\
4,0\end{array}$ & $\begin{array}{r}12,1 \\
13,9 \\
6,8 \\
7,5 \\
7,1\end{array}$ \\
\hline
\end{tabular}

\begin{tabular}{|c|c|c|}
\hline & CRECIMIENTO & MIGRATORIO \\
\hline & \multicolumn{2}{|c|}{ Cifras absolutas } \\
\hline 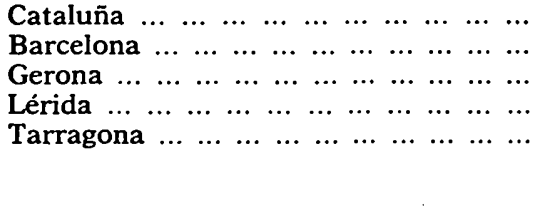 & $\begin{array}{r}469.806 \\
479.613 \\
10.370 \\
-11.789 \\
-8.388 \\
\text { Porce }\end{array}$ & $\begin{array}{r}720.480 \\
649.610 \\
39.077 \\
-11.876 \\
43.669 \\
\text { ntajes }\end{array}$ \\
\hline 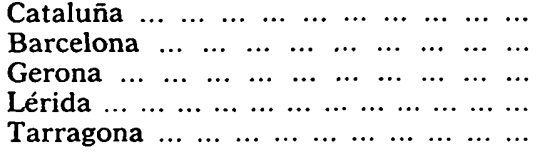 & $\begin{array}{r}14,5 \\
21,5 \\
3,1 \\
-3,6 \\
-2,4\end{array}$ & $\begin{array}{r}18,4 \\
22,6 \\
11,1 \\
-3,5 \\
12,0\end{array}$ \\
\hline
\end{tabular}


El crecimiento vegetativo medio en el conjunto de España fue del 12 por 100 en el primer decenio considerado y del 12,8 en el segundo, mientras que el crecimiento migratorio fue negativo. En todo caso, el incremento vegetativo - diferencia entre nacimientos y defunciones - fue claramente inferior al nacional en el primer decenio, situación que mejora mucho para Barcelona en el segundo decenio, ya que presenta una tasa superior a la nacional y ello repercute en la tasa de Cataluña en su conjunto, compensando los bajos incrementos de las restantes Provincias catalanas.

En cuanto al crecimiento migratorio, es positivo y con tasas muy elevadas para el conjunto de Cataluña y para Barcelona especialmente, y para Gerona y Tarragona en el segundo decenio. No así para Lérida, con saldo migratorio negativo en los dos decenios, y para Tarragona en el primero de ellos.

En el primer decenio el crecimiento vegetativo tiene más importancia que el migratorio para Gerona, Lérida y Tarragona, situación que cambia de signo para Gerona y Tarragona en el segundo decenio. En todo caso, es evidente que el incremento de la población de Cataluña obedece primordialmente al crecimiento migratorio y que ello se da especialmente con referencia a la Provincia de Barcelona y, por ende, dado el peso demográfico de esta Provincia, sobre el conjunto de Cataluña.

Cataluña absorbió en el primer decenio el 27,9 por 100 del incremento de la población española, y en el segundo decenio esta tasa se elevó al 35,3, es decir, que la tercera parte del aumento de población en el país fue absorbido por Cataluña.

\section{LAS TASAS DE NATALIDAD, MORTALIDAD Y NUPCIALIDAD}

La tasa de natalidad, que en el conjunto de España presenta una ligera tendencia decreciente, se ofrece ligeramente creciente para Cataluña y las Provincias individualmente consideradas, con la salvedad de Lérida, aunque partiendo de cotas más bajas en los veinte años que comprenden los dos decenios considerados. En el año 1971 la tasa de Cataluña es del 19,7 por 1.000 habitantes, muy similar al 19,5 del conjunto nacional, y es superada por la de Barcelona con el 20,6. En el mismo año las tasas de las trés Provincias restantes se sitúan en el 15,3 para Lérida, el 16,8 para Gero- 
na y el 17,5 para Tarragona. Es marcada la desigualdad entre las Provincias catalanas en cuanto a la natalidad se refiere.

La tasa de mortalidad ha decrecido en Cataluña, al igual que en el conjunto del país, situándose en el 8,9 por 1.000 habitantes para ambas áreas territoriales en 1971. En la Provincia de Barcelona el descenso es más acusado y llega al 8,3 para el año citado. En las otras tres Provincias la disminución ha sido menos acentuada y se mantienen en 1971 tasas del 10,1 para Lérida, 10,5 para Gerona y 11,4 para Tarragona.

Mientras que la tasa de nupcialidad se ha mantenido prácticamente inalterable en el período 1951-1970, en Cataluña el proceso ha sido descendente, aunque la tasa de Cataluña en 1971 ha sido del 7,5 por 1.000 habitantes, superior, al igual que la de Barcelona, cifrada en el 7,7, a la del conjunto de España, establecida en el 7,4. La tasa de Lérida se fija en el 6,4, la de Gerona en el 7,1 y la de Tarragona en el 7,0 todas ellas en 1971.

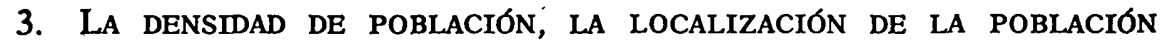
SEGÚN EL TAMAÑo DEMOGRAFICO DE LOS MUNICIPIOS Y LAS TASAS DE URBANIZACIÓN

La densidad de población de España se fijó en 1970 en 67 habitantes por kilómetro cuadrado. La de Cataluña, muy superior, es de 160. La Provincia de Barcelona, mucho más densamente poblada, ofrece una densidad de 508, Gerona y Tarragona, costeras, dan una densidad de 70 y 69, respectivamente, todavía superior a la nacional. Tan sólo Lérida se sitúa solamente en 29.

Mientras que en el conjunto de España el 43 por 100 de sus habitantes residen en Municipios mayores de 50.000 habitantes, tal tasa se eleva al 59,3 para Cataluña y al 70,4 para la Provincia de Barcelona. Por el contrario, en las tres Provincias restantes más del 50 por 100 de sus habitantes residen en Municipios de hasta 10.000 habitantes, si bien se nota una tendencia hacia la concentración en Municipios de mayor entidad demográfica en Tarragona y Lérida.

Si tenemos en cuenta la población residente en Municipios mayores de 20.000 habitantes, mientras que representa el 55 por 100 de sus habitantes para el conjunto de España, en Cataluña se eleva al 69,9 y en la Provincia de Barcelona al 82,1. Las tasas de las res- 
tantes Provincias, inferiores, son del 42,4 para Tarragona, el 25,6 para Lérida y tan sólo el 22,5 para Gerona.

\section{LA NATURALEZA DE LA POBLACIÓN Y LAS MIGRACIONES INTERIORES}

Según las «Estadísticas de la población de España deducidas del Padrón Municipal de Habitantes del año 1865", publicadas por el Instituto Nacional de Estadística, la distribución de la población por su naturaleza para España, Cataluña y las cuatro Provincias catalanas fue, expresada en porcentajes, la siguiente:

\begin{tabular}{|c|c|c|c|c|c|}
\hline & \multicolumn{5}{|c|}{ NACIDOS } \\
\hline & $\begin{array}{l}\text { En el mis- } \\
\text { mo Munic. } \\
\text { de resid. }\end{array}$ & $\begin{array}{c}\text { En otros } \\
\text { Municipios } \\
\text { de la Prov. }\end{array}$ & $\begin{array}{l}\text { En otras } \\
\text { Provincias }\end{array}$ & $\begin{array}{c}\text { En el } \\
\text { extranjero }\end{array}$ & $\begin{array}{c}\text { No } \\
\text { consta }\end{array}$ \\
\hline $\begin{array}{cccccc}\text { España } & \ldots & \ldots & \ldots & \ldots & \ldots\end{array}$ & 63 & 15 & 21 & 1 & - \\
\hline $\begin{array}{cccccc}\text { Cataluña } & \ldots & \ldots & \ldots & \ldots & \ldots\end{array}$ & 43 & 14 & 41 & 1 & 1 \\
\hline $\begin{array}{ccccc}\text { Barcelona } & \ldots & \ldots & \ldots & \ldots\end{array}$ & 41 & 11 & 46 & 1 & 1 \\
\hline $\begin{array}{cccccc}\text { Gerona } & \ldots & \ldots & \ldots & \ldots & \ldots\end{array}$ & 44 & 27 & 25 & 1 & 3 \\
\hline $\begin{array}{lllllll}\text { Lérida } & \ldots & \ldots & \ldots & \ldots & \ldots & \ldots\end{array}$ & 55 & 22 & 23 & - & - \\
\hline $\begin{array}{ccccc}\text { Tarragona } & \ldots & \ldots & \ldots & \ldots\end{array}$ & 56 & 15 & 28 & 1 & - \\
\hline
\end{tabular}

El porcentaje de nacidos en el mismo Municipio de residencia, que es de 63 habitantes de cada 100 para el conjunto nacional, resulta notoriamente inferior para Cataluña, Barcelona y Gerona; tan sólo Lérida y Tarragona tienen tasas cercanas, aunque inferiores. Ello indica, en principio, que Cataluña está muy afectada por los movimiento migratorios, en especial los procedentes de otras Provincias, ya que mientras que los habitantes nacidos en otras Pro vincias son sólo el 21 por 100 en el conjunto nacional, en Cataluña se elevan al 41 y en la Provincia de Barcelona al 46, y aun las restantes Provincias presentan tasas superiores a la nacional.

Es de notar, sin embargo, que ni Cataluña en conjunto, ni Barcelona, ni Tarragona están más afectadas que España en conjunto, en cuanto a la atracción de inmigrantes procedentes de otros Municipios de la misma Provincia, ya que no rebasan la tasa media nacional. Tan sólo en Lérida y Gerona esta corriente tiene una fuerza mayor. La inmigración procedente del extranjero es muy similar a la nacional, a excepción de Lérida.

En resumen, Cataluña se encuentra fuertemente afectada por 
un movimiento inmigratorio tenaz y persistente que permite abiertamente el crecimiento de su población, muy acusado para la Provincia de Barcelona $\mathrm{y}$, por ende, para el conjunto de Cataluña, y más moderado en las tres restantes Provincias.

En el período 1966-1970, las migraciones interiores españolas, que han afectado a Cataluña, han supuesto la inmigración de 594.649 personas, distribuidas por Provincias en esta forma:

\begin{tabular}{|c|c|c|}
\hline & Personas & Porcentajes \\
\hline $\begin{array}{lllllllllll}\text { Barcelona } & \ldots & \ldots & \ldots & \ldots & \ldots & \ldots & \ldots & \ldots & \ldots & \ldots \\
\text { Gerona } & \ldots & \ldots & \ldots & \ldots & \ldots & \ldots & \ldots & \ldots & \ldots & \ldots \\
\text { Lérida } & \ldots & \ldots & \ldots & \ldots & \ldots & \ldots & \ldots & \ldots & \ldots & \ldots \\
\text { Tarragona } & \ldots & \ldots & \ldots & \ldots & \ldots & \ldots & \ldots & \ldots & \ldots\end{array}$ & $\begin{array}{r}492.640 \\
44.243 \\
22.836 \\
34.930\end{array}$ & $\begin{array}{r}82,9 \\
7,4 \\
3,8 \\
5,9\end{array}$ \\
\hline
\end{tabular}

La inmigración media anual en el período de referencia ha sido de algo más de 118.000 personas. Cuatro de cada cinco se han establecido en la Provincia de Barcelona, siendo muy reducida, aunque positiva, la fuerza de atracción de las otras tres Provincias. A la Provincia de Barcelona, además del fuerte contingente de inmigrantes procedentes de la Provincia, que supone el 40 por $100 \mathrm{del}$ total, llegan gentes que provienen, ante todo, de Andalucía, y en un segundo término, de Extremadura, las dos Castillas, Galicia y León, Aragón y, en último término, de las restantes Provincias catalanas. Gerona se halla afectada en su inmigración por gentes que provienen de la propia Provincia y de Andalucía y Extremadura principalmente. El principal contingente de inmigrantes de Lérida procede de la misma Provincia y, en segundo término, de Andalucía, Aragón y la Provincia de Barcelona. El área de atracción de Tarragona es más amplio que la de las dos Provincias citadas con anterioridad; aparte de la inmigración de la propia Provincia, sus inmigrantes extraprovinciales provienen de Andalucía, de la Provincia de Barcelona, Lérida, Aragón, Castilla la Nueva y Extremadura y Valencia.

5. LA ESTRUCTURA DE LA POBLACIÓN POR SEXO Y EDAD

Y LA COMPOSICIÓN DE LA POBLACIÓN ECONÓMICAMENTE ACTIVA

Existe en Cataluña, como en el resto de España, un predominio del sexo femenino, con el 51,3 por 100 de mujeres en 1970 , muy si- 
milar al 51,2 del conjunto de España; Barcelona registra el 51,6 y Gerona y Tarragona el 50,8 y el 50,6, respectivamente. Tan sólo Lérida da, a lo largo de los años 1950 a 1970, un porcentaje menor de mujeres, cifrado en el 49,9 en 1970.

La distribución por edades presenta el siguiente detalle para cada Provincia y el conjunto de Cataluña en el año 1970, según el Censo de Población de dicho año y expresado en porcentajes:

\begin{tabular}{|c|c|c|c|}
\hline & $\begin{array}{c}\text { Menores } \\
\text { de } 15\end{array}$ & De 15 a 64 & De 65 y más \\
\hline $\begin{array}{lllllllllll} & \ldots & \ldots & \ldots & \ldots & \ldots & \ldots & \ldots & \ldots & \ldots & \ldots\end{array}$ & 27,8 & 62,5 & 9,7 \\
\hline 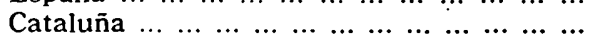 & 25,6 & 64,5 & 9,9 \\
\hline $\begin{array}{llllllllllll}\text { Barcelona } & \ldots & \ldots & \ldots & \ldots & \ldots & \ldots & \ldots & \ldots & \ldots & \ldots & \ldots\end{array}$ & 26,1 & 64,6 & 9,3 \\
\hline 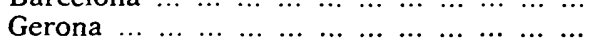 & $\begin{array}{l}20,1 \\
23,1\end{array}$ & 65,0 & 11,9 \\
\hline 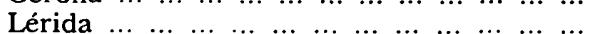 & 248 & 63.0 & 12.2 \\
\hline $\begin{array}{ccccccccccc}\text { Tarragona } & \ldots & \ldots & \ldots & \ldots & \ldots & \ldots & \ldots & \ldots & \ldots & \ldots\end{array}$ & 24,8 & 62,4 & 12,8 \\
\hline
\end{tabular}

La distribución no difiere grandemente con respecto al conjunto de España, siendo de señalar la mayor proporción de personas jóvenes en la Provincia de Barcelona'y en el conjunto de Cataluña, y la de viejos en las otras tres Provincias, mientras que el grupo intermedio supera al nacional, con la excepción de Tarragona.

La población económicamente activa presenta, por sexos, esta distribución, en porcentajes:

\begin{tabular}{|c|c|c|c|}
\hline & Varones & Mujeres & En conj. \\
\hline $\begin{array}{lllllllllll}\ldots & \ldots & \ldots & \ldots & \ldots & \ldots & \ldots & \ldots & \ldots & \ldots & \ldots\end{array}$ & 57 & 13 & 35 \\
\hline $\begin{array}{llllllllllll}\text { Cataluña } & \ldots & \ldots & \ldots & \ldots & \ldots & \ldots & \ldots & \ldots & \ldots & \ldots & \ldots\end{array}$ & 60 & 18 & 39 \\
\hline $\begin{array}{llllllllllll}\text { Barcelona } & \ldots & \ldots & \ldots & \ldots & \ldots & \ldots & \ldots & \ldots & \ldots & \ldots & \ldots\end{array}$ & 60 & 19 & 39 \\
\hline $\begin{array}{llllllllllllll}\text { Gerona } & \ldots & \ldots & \ldots & \ldots & \ldots & \ldots & \ldots & \ldots & \ldots & \ldots & \ldots & \ldots\end{array}$ & 63 & 18 & 40 \\
\hline $\begin{array}{lllllllllllll} & \\
\text { Lérida } & \ldots & \ldots & \ldots & \ldots & \ldots & \ldots & \ldots & \ldots & \ldots & \ldots & \ldots & \ldots \\
\end{array}$ & 60 & 11 & 36 \\
\hline $\begin{array}{cccccccccccc}\text { Tarragona } & \ldots & \ldots & \ldots & \ldots & \ldots & \ldots & \ldots & \ldots & \ldots & \ldots & \ldots\end{array}$ & 60 & 13 & 36 \\
\hline
\end{tabular}

El porcentaje de población activa es superior en Cataluña al conjunto de España, de manera más acentuada en Barcelona y Gerona y más moderadamente en Lérida y Tarragona. Igualmente es superior la población activa masculina, similar en toda la Región, con la excepción de Gerona, que ofrece la máxima. También destaca la población activa femenina en Barcelona y Gerona, mientras que en Tarragona o Lérida se mantiene al mismo nivel o a nivel inferior al del conjunto nacional. 
ción de la actividad industrial de Tarragona a la del conjunto de España y la tasa inferior, en este orden, que ofrece Lérida. Es de señalar, además, la actividad agraria predominante en Lérida, y todavía alta en Tarragona, así como la tasa de servicios de Barcelona, más reducida que la nacional en las otras tres Provincias.

Cataluña da, de otra parte, una alta tasa de trabajadores asalariados, especialmente en Barcelona, y de trabajadores autónomos en las otras tres Provincias. La actividad familiar destaca todavía en Lérida. $\mathrm{Y}$ consiguientemente es muy alta la disponibilidad de obreros cualificados o especializados en la Provincia de Barcelona y en la de Gerona, y de empresarios agrarios sin asalariados en Lérida, Tarragona y Gerona.

Ha quedado patente el peso demográfico de Barcelona, cuyas características enmarcan y condicionan al conjunto de Cataluña. Gerona y Tarragona, Provincias costeras, se incorporan, a menor nivel, hacia las metas de Barcelona, y Lérida, interior, queda todavía reflejando una economía y una demografía primordialmente agrarias.

\section{LA ACTIVIDAD ECONOMICA: AGRARIA, INDUSTRIAL Y DE SERVICIOS}

En cuanto a la actividad agraria, hemos de destacar los datos de mayor significación. La superficie agrícola de Cataluña es de 3,2 millones de Has., el 6,3 por 100 de la superficie agrícola nacional. La superficie labrada se fija en 1,16 millones de Has., de las que 910.000 son de secano y 257.000 de regadío, que suponen, respectivamente, el 5,2 y el 11,5 por 100 del secano o del regadío en el conjunto nacional. De cada 100 Has. en Cataluña, 78 se cultivan en secano y 22 en regadío; este último predomina en Tarragona - delta del Ebro- y Lérida - Llanos de Urgel- El parque de maquinaria representa el 14 por 100 de los tractores del país, el 16 de los motocultores, el 11 de los motores y el 10 y el 9 de trilladoras y cosechadoras. Mientras que el número de hectáreas labradas por tractor es de 76 para el conjunto de España, en Cataluña es de 32, con los valores mínimos de 23 y 22 para Barcelona y Gerona.

Cataluña dispone del 6,4 por 100 del ganado vacuno del país, del 4 por 100 del lanar y del 15 por 100 del caprino. Destaca el volumen del ganado porcino, que representa el 24 por 100 del total 
nacional. El ganado caballar, mular y asnal suponen, respectivamente, el $4,7,3,9$ y 1,3 por 100 del total nacional.

La población activa agraria se cifraba en el año 1969 en 233.481 personas, de las que solamente el 20 por 100 eran trabajadores por cuenta ajena y el 80 trabajadores autónomos y empresarios.

La producción final agraria tenía en 1969 un valor de $33.791 \mathrm{mi}$ llones de pesetas, que se distribuían en esta forma:

\begin{tabular}{|c|c|}
\hline & $\begin{array}{l}\text { Millones } \\
\text { de pesetas }\end{array}$ \\
\hline Producción final agrícola ... ........ & 19.796 \\
\hline Producción final ganadera $\ldots \ldots \ldots \ldots$ & 13.300 \\
\hline Producción final forestal $\ldots \ldots \ldots \ldots \ldots$ & 695 \\
\hline
\end{tabular}

En la producción agrícola destacan, en primer término, las hortalizas, que representan los dos tercios del total, los cereales y las plantas industriales. En ganadería, el 60 por 100 del valor total se asigna a ganado para abasto, seguido de la producción de huevos y leche. En la forestal destacan las maderas.

En la actividad agrícola destacan Lérida y Tarragona, y en la ganadera y forestal, Gerona y Barcelona.

En 1970 la pesca de litoral y altura alcanza 23.475 Tm., que representa el 3,4 por 100 del total nacional. Tarragona, Gerona y Barcelona, por este orden, son las que dan las capturas pesqueras. Su valor es de 615 millones de pesetas.

La producción industrial se eleva en 1969 a 525.962 millones de pesetas, que representa el 25,4 por 100 de la producción nacional. Frente al valor de facturación que representa el valor de la producción industrial, el producto industrial bruto o valor añadido bruto ha sido en dicho año de 183.670 millones de pesetas.

El valor de la producción industrial se distribuye por Provincias en esta forma:

\begin{tabular}{|c|c|c|}
\hline & $\begin{array}{c}\text { Millones } \\
\text { de pesetas }\end{array}$ & Porcentajes \\
\hline 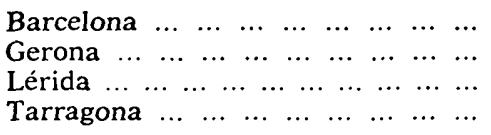 & $\begin{array}{r}428.439 \\
36.983 \\
21.196 \\
39.344\end{array}$ & $\begin{array}{r}81,5 \\
7,0 \\
4,0 \\
7,5\end{array}$ \\
\hline
\end{tabular}


lo que da idea clara de la muy fuerte concentración industrial en la Provincia de Barcelona.

La distinta participación de los sectores industriales en el producto industrial bruto en el año 1969, distribuido por Provincias y comparado con el conjunto de Cataluña y nacional, es como sigue:

\begin{tabular}{|c|c|c|c|c|c|c|}
\hline & \multicolumn{6}{|c|}{ PORCENTAJES } \\
\hline & España & Catal. & Barcel. & Gerona & Lérida & Tarrag. \\
\hline Minería & 2.8 & 0,8 & 0,8 & 1,1 & 1,8 & 0,6 \\
\hline Alimentación, bebidas y tabacos. & 11.5 & 8,8 & 6,1 & 12,3 & 21,3 & 31,3 \\
\hline 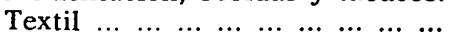 & 5.5 & 15,9 & 17,4 & 16,1 & 4,5 & 3,9 \\
\hline Cuero, calzado y confección ... & 7,1 & 6,3 & 6,5 & 5,3 & 5,3 & 5,5 \\
\hline Madera y corcho $\ldots \ldots \ldots \ldots \ldots$ & 4.1 & 3,4 & 2,9 & 8,0 & 4,5 & 5,1 \\
\hline Papel, prensa y artes gráficas ... & 3.7 & 4,3 & 4,5 & 5,6 & 3,3 & 1,9 \\
\hline Químicas y derivados del petró & & & & & & \\
\hline leo y carbón $\ldots \ldots \ldots \ldots \ldots$ & 10,9 & 14,0 & 14,5 & 12,7 & 2,5 & 15,8 \\
\hline Cerámica, vidrio y cemento ........ & 4.3 & 3,8 & 3,8 & 3,1 & 4,7 & 3,5 \\
\hline Metálicas básicas $\ldots \ldots \ldots \ldots$ & 6,1 & 1,5 & 1,7 & 0,4 & 0,1 & 0,2 \\
\hline Transformados metálicos $\ldots \ldots$ & 23,6 & 26,7 & 29,2 & 15,0 & 13,3 & 13,7 \\
\hline Edificación y obras públicas ... & 14.7 & 10,4 & 9,6 & 15,6 & 16,3 & 12,2 \\
\hline Agua, gas y electricidad $\ldots \ldots \ldots$ & 5,7 & 4,1 & 3,0 & 4,8 & 22,4 & 6,3 \\
\hline
\end{tabular}

No aparece Cataluña como una Región predominantemente minera. En tres sectores industriales es evidente el predominio catalán: textil, químico y de transformados metálicos; la industria textil se asienta principalmente en las Provincias de Barcelona y Gerona; la industria química, más generalizada, se extiende, además, a Tarragona; la de transformados metálicos se limita a las dos que resaltan en la textil. A segundo nivel también destaca Cataluña en la industria del papel, que se ubica en Gerona y Barcelona igualmente. La actividad industrial de preparación y fabricación de bebidas y de elaboración de tabacos tiene importancia superior a la nacional, no en el conjunto de Cataluña, pero sí en las Provincias de Gerona, Lérida y Tarragona. De igual forma, las industrias de la madera y corcho destacan en Gerona - primera zona corchera nacional-y en las Provincias de Lérida y Tarragona; y el sector de cerámica, vidrio y cemento resalta preponderantemente en Lérida. Gerona y Lérida sobresalen por las obras públicas que en estas Provincias se realizan y, finalmente, Lérida destaca también por ser una de las Provincias de mayor producción de energía hidroeléctrica.

No se dispone de datos referentes a todos los subsectores del 
sector servicios. El turismo es una actividad moderna, que ha registrado un impulso creciente en los últimos quince años. En el año 1971 han visitado Cataluña 8.663.000 turistas extranjeros, lo que equivale a la tercera parte de los turistas entrados en el país. La Costa Brava y la Costa Dorada han llegado a constituir dos zonas turísticas litorales perfectamente definidas, e igualmente destaca Cataluña por su turismo de invierno, aún no tan desarrollado como es de desear.

De la misma manera que ocurre respecto al turismo receptivo nacional, el 79 por 100 de los turistas entrados lo hacen por carretera, el 12 por vía aérea, el 6,6 por ferrocarril y el 2,5 por los puertos marítimos. Estos turistas se alojan en establecimientos hoteleros y campings y en un número creciente de apartamentos, chalés, villas y complejos turísticos, que constituyen la infraestructura de esta actividad, siempre creciente en Cataluña.

En diciembre de 1971 existían en Cataluña 2.122 establecimientos hoteleros, que suponen el 24,6 por 100 de todos los españoles, con una capacidad de 140.891 plazas, el 23 por 100 del total. Su distribución provincial es ésta:

\begin{tabular}{|c|c|c|c|c|}
\hline & Hoteles & Plazas & Turistas & $\begin{array}{l}\text { Pernoc- } \\
\text { taciones }\end{array}$ \\
\hline $\begin{array}{lllllllllll}\text { Barcelona } & \ldots & \ldots & \ldots & \ldots & & \ldots & & & & \\
\text { Gerona } & \ldots & \ldots & \ldots & \ldots & \ldots & \ldots & \ldots & \ldots & \ldots \\
\text { Lérida } & \ldots & \ldots & \ldots & \ldots & \ldots & \ldots & \ldots & \ldots & \ldots & \ldots \\
\text { Tarragona } & \ldots & \ldots & \ldots & \ldots & \ldots & \ldots & \ldots & \ldots & \ldots \\
\text { Tarran } & \ldots & \ldots & \ldots & \ldots\end{array}$ & $\begin{array}{l}889 \\
939 \\
149 \\
145\end{array}$ & $\begin{array}{r}57.825 \\
65.202 \\
6.830 \\
11.034\end{array}$ & $\begin{array}{r}638.868 \\
676.696 \\
44.727 \\
179.506\end{array}$ & $\begin{array}{r}5.388 .992 \\
4.499 .244 \\
459.603 \\
915.452\end{array}$ \\
\hline
\end{tabular}

\begin{tabular}{|c|c|c|}
\hline & $\begin{array}{c}\text { Plazas } \\
\text { por hotel }\end{array}$ & $\begin{array}{l}\text { Pernocta- } \\
\text { ciones por } \\
\text { turista }\end{array}$ \\
\hline $\begin{array}{llllllllllll}\text { Barcelona } & \ldots & \ldots & \ldots & \ldots & \ldots & \ldots & \ldots & \ldots & \ldots & \ldots & \ldots \\
\text { Gerona } & \ldots & \ldots & \ldots & \ldots & \ldots & \ldots & \ldots & \ldots & \ldots & \ldots & \ldots \\
\text { Lérida } & \ldots & \ldots & \ldots & \ldots & \ldots & \ldots & \ldots & \ldots & \ldots & \ldots & \ldots \\
\text { Tarragona } & \ldots & \ldots & \ldots & \ldots & \ldots & \ldots & \ldots & \ldots & \ldots & \ldots & \ldots \\
\text { Tarraten } & \ldots & \ldots & \ldots & \ldots & \ldots & \ldots\end{array}$ & $\begin{array}{l}65 \\
69 \\
45 \\
76\end{array}$ & $\begin{array}{r}8 \\
7 \\
10 \\
5\end{array}$ \\
\hline
\end{tabular}

Quedan determinadas la capacidad media por hotel y la estancia media por turista. La primera indica que los hoteles de mayor tamaño se hallan en la Provincia de Tarragona, siguiendo a continuación los de Gerona y Barcelona, y los más pequeños se hallan en la Provincia de Lérida. Los 1.539 .797 turistas que los utilizaron 
causaron 11.263.291 pernoctaciones. La estancia media por turista mayor correspondió a Lérida y la menor a Tarragona.

De otra parte, funcionaron en Cataluña, durante el expresado año, 230 campings, que representan casi la mitad de los existentes en España. Su capacidad es de 115.320 plazas.

No se dispone de datos sobre los apartamentos y otros alojamientos extrahoteleros, si bien comparando la cifra de turistas entrados con la de usuarios de los establecimientos hoteleros se advierte que debe ser mucho mayor el número de los que residen en los apartamentos y otros alojamientos extrahoteleros durante sus viajes por España.

Con referencia a los transportes, tengamos presente que el parque de vehículos en Cataluña en el año 1971 era de 1.039.092, el 21,5 por 100 del total de España. El 80 por 100 corresponde a la Provincia de Barcelona. La longitud de las carreteras, autovías y autopistas de Cataluña era, en dicho año, de $10.235 \mathrm{kms}$., el 7,3 por 100 de la red nacional. Al transporte por carretera se dedican 112.604 vehículos de motor mecánico, el 90 por 100 de ellos dedicados al transporte de mercancías. La red de carreteras resulta insuficiente para Cataluña, pues se dispone tan sólo de $197 \mathrm{kms}$. de carretera por 100.000 habitantes, cuando para el conjunto nacional tal disponibilidad alcanza a $410 \mathrm{kms}$. por 100.000 habitantes. Igualmente es reducida la red ferroviaria.

Con respecto al transporte maritimo, es de señalar que en el puerto de Barcelona entraron 9.143 buques mercantes en el año 1971 , con 798.000 pasajeros y nueve millones de toneladas de mercancías transportadas. En Tarragona entraron 2.471 buques, con casi cinco millones de toneladas de mercancías y un reducido número de pasajeros. El tráfico de ambos puertos conjuntamente representa el 10 por 100 del tráfico marítimo nacional.

El tráfico aéreo ha tenido un marcado incremento en los últimos años. En el aeropuerto de Barcelona, en el año 1970, entraron y salieron 61.232 aeronaves, con 2,8 millones de pasajeros y 31.000 toneladas de mercancía. El número de aeronaves entradas y salidas en el aeropuerto de Gerona fue de 12.160 y en el de Reus de 1.132. En conjunto, pues, los tres aeropuertos registraron un movimiento de 74.524 aeronaves, con 3.556 .654 pasajeros transportados, que representa el 16,6 por 100 del tráfico aéreo nacional. 


\section{LA RENTA Y EL CONSUMO}

Las rentas salariales se elevaron en el año 1969 a 168.900 millones de pesetas, según las estimaciones del Banco de Bilbao, que equivalen al 21,1 por 100 de la renta salarial nacional. Por sectores de actividad se distribuyen en esta forma:

\begin{tabular}{|c|c|c|}
\hline & $\begin{array}{l}\text { Millones } \\
\text { de pesetas }\end{array}$ & $\begin{array}{c}\text { Porcentajes } \\
\text { sobre } \\
\text { el total } \\
\text { nacional }\end{array}$ \\
\hline $\begin{array}{lllllllll} & \ldots & \ldots & \ldots & \ldots & \ldots & \ldots & \ldots & \ldots\end{array}$ & $3.557,2$ & 5,47 \\
\hline $\begin{array}{llllllll}\text { Agricultura, } \text { ganadería } \text { y silvicultura } & \ldots & \ldots & \ldots & \ldots & \ldots & \ldots\end{array}$ & $2.939,1$ & 5,35 \\
\hline 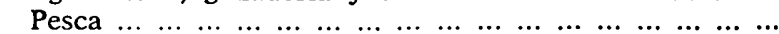 & 618,1 & 6,07 \\
\hline $\begin{array}{lllllllllllllll}\text { Sector industrial } & \ldots & \ldots & \ldots & \ldots & \ldots & \ldots & \ldots & \ldots & \ldots & \ldots & \ldots & \ldots & \ldots & \ldots\end{array}$ & $98.255,1$ & 26,21 \\
\hline 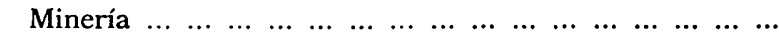 & $1.076,4$ & 7,39 \\
\hline 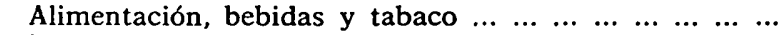 & $5.442,0$ & 18,57 \\
\hline $\begin{array}{lllllllllllllllll}\operatorname{Textil} & \ldots & \ldots & \ldots & \ldots & \ldots & \ldots & \ldots & \ldots & \ldots & \ldots & \ldots & \ldots & \ldots & \ldots & \ldots & \ldots\end{array}$ & $14.902,7$ & 72,51 \\
\hline 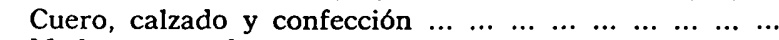 & $4.003,1$ & 21,94 \\
\hline 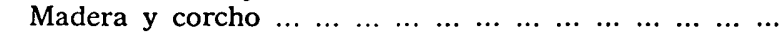 & $3.063,5$ & 22,02 \\
\hline 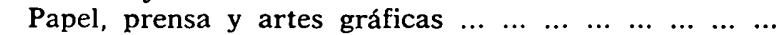 & $4.389,4$ & 31,41 \\
\hline Químicas y derivados del petróleo y carbón ... ......... & $11.045,5$ & 35,47 \\
\hline $\begin{array}{lllllllllll}\text { Cerámica, vidrio } & \text { y } \\
\text { cemento } & \ldots & \ldots & \ldots & \ldots & \ldots & \ldots & \ldots & \ldots & \ldots\end{array}$ & $3.547,6$ & 23,15 \\
\hline $\begin{array}{lllllllllllll}\text { Metálicas básicas } & \ldots & \ldots & \ldots & \ldots & \ldots & \ldots & \ldots & \ldots & \ldots & \ldots & \ldots & \ldots\end{array}$ & $1.455,8$ & 6,64 \\
\hline $\begin{array}{lllllllllll}\text { Transformados metálicos } & \ldots & \ldots & \ldots & \ldots & \ldots & \ldots & \ldots & \ldots & \ldots & \ldots\end{array}$ & $32.837,1$ & 29,40 \\
\hline $\begin{array}{llllllllll}\text { Edificación } \mathrm{y} \text { obras públicas } & \ldots & \ldots & \ldots & \ldots & \ldots & \ldots & \ldots & \ldots & \ldots\end{array}$ & $13.932,5$ & 18,81 \\
\hline 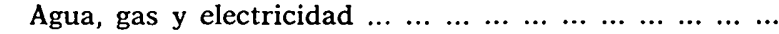 & $2.559,5$ & 25,14 \\
\hline $\begin{array}{lllllllllllllll}\operatorname{Sector} \operatorname{servicios} & \ldots & \ldots & \ldots & \ldots & \ldots & \ldots & \ldots & \ldots & \ldots & \ldots & \ldots & \ldots & \ldots\end{array}$ & $67.087,7$ & 18,43 \\
\hline $\begin{array}{llllllllllllllll}\text { Comercio } & \ldots & \ldots & \ldots & \ldots & \ldots & \ldots & \ldots & \ldots & \ldots & \ldots & \ldots & \ldots & \ldots & \ldots & \ldots\end{array}$ & $11.062,8$ & 23,82 \\
\hline 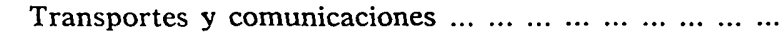 & $12.683,0$ & 18,83 \\
\hline $\begin{array}{lllllllllllll}\text { Ahorro, banca } \mathrm{y} \text { seguros } & \ldots & \ldots & \ldots & \ldots & \ldots & \ldots & \ldots & \ldots & \ldots & \ldots\end{array}$ & $6.768,8$ & 23,03 \\
\hline 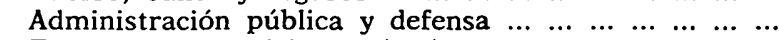 & $14.203,2$ & 25,28 \\
\hline 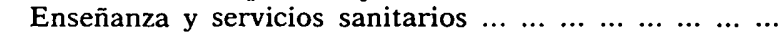 & $6.959,7$ & 8,34 \\
\hline 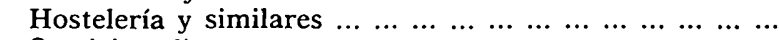 & $11.128,2$ & 19,51 \\
\hline $\begin{array}{lllllllllllll}\text { Servicios } \operatorname{diversos} & \ldots & \ldots & \ldots & \ldots & \ldots & \ldots & \ldots & \ldots & \ldots & \ldots & \ldots & \ldots\end{array}$ & $4.282,0$ & 17,77 \\
\hline
\end{tabular}

Los porcentajes anotados representan para cada sector o rama de actividad lo que la respectiva actividad en Cataluña representa sobre el total nacional, tomando como base las remuneraciones del personal asalariado. De ellos se deduce, en primer término, el escaso peso de la actividad de la Región en el sector primario y el relevante en el sector industrial, en el que supone más de la quinta parte de la actividad nacional, y en el sector de servicios, en el que representa más de la sexta parte.

Entre las ramas de la actividad industrial destacan, por sus mayores porcentajes $\mathrm{y}$, consiguientemente, por su importancia prepon- 
derante en la Región respecto al total nacional, la industria textil, con casi las tres cuartas partes del total nacional; la industria química, con la tercera parte, y acercándose a este valor la de papel, prensa y artes gráficas, a las que siguen, con más del 20 por 100 , transformados metálicos; agua, gas y electricidad; cerámica, vidrio y cemento; madera y corcho, y cuero, calzado y confección; y con cerca de la quinta parte, edificación y obras públicas, y alimentación, bebidas y tabaco.

En el sector de servicios son de señalar, con la quinta parte del total, la Administración pública y defensa, acercándose a este nivel el comercio y el ahorro, banca y seguros, y entre la sexta y la cuarta parte se hallan situadas la hostelería, los transportes y comunicaciones y los demás servicios.

Las rentas de otros factores se elevan a 193.190 millones de pesetas, equivalentes al 19 por 100 del total nacional, y se discriminan así:

\begin{tabular}{|c|c|c|}
\hline & $\begin{array}{l}\text { Millones } \\
\text { de pesetas }\end{array}$ & $\begin{array}{c}\text { Porcentajes } \\
\text { sobre } \\
\text { el total } \\
\text { nacional }\end{array}$ \\
\hline $\begin{array}{lllllllllll}\ldots & \ldots & \ldots & \ldots & \ldots & \ldots & \ldots & \ldots & \ldots & \ldots\end{array}$ & $54.726,5$ & 21,33 \\
\hline 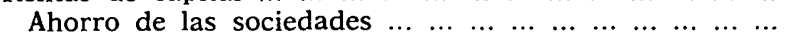 & $17.476,6$ & 23,67 \\
\hline 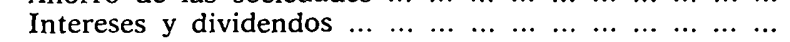 & $20.547,9$ & 20,77 \\
\hline 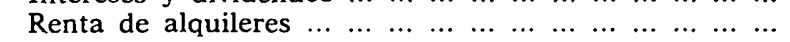 & $16.702,0$ & 19,93 \\
\hline $\begin{array}{lllllllllllllll}\operatorname{Rentas} \operatorname{mixtas} & \ldots & \ldots & \ldots & \ldots & \ldots & \ldots & \ldots & \ldots & \ldots & \ldots & \ldots & \ldots & \ldots & \ldots\end{array}$ & $85.251,0$ & 16,59 \\
\hline 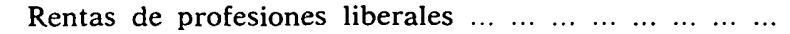 & $16.469,9$ & 21,57 \\
\hline 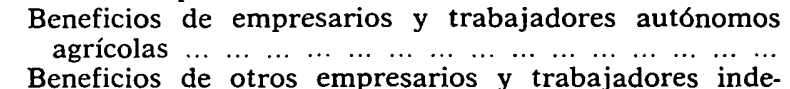 & $20.411,5$ & 10,52 \\
\hline $\begin{array}{lllllllllllllll}\text { pendientes } & \ldots & \ldots & \ldots & \ldots & \ldots & \ldots & \ldots & \ldots & \ldots & \ldots & \ldots & \ldots & \ldots & \ldots\end{array}$ & $48.369,6$ & 19,88 \\
\hline Resto de ingresos $\begin{array}{llllllllllllll} & \ldots & \ldots & \ldots & \ldots & \ldots & \ldots & \ldots & \ldots & \ldots & \ldots & \ldots & \ldots & \ldots\end{array}$ & $53.213,0$ & 21,60 \\
\hline Cuotas Seguridad Social y Clases Pasivas .............. & $36.730,0$ & 20,58 \\
\hline 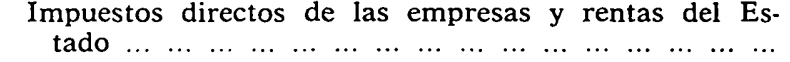 & $16.483,0$ & 24,28 \\
\hline
\end{tabular}

Puede observarse la importancia de los conceptos vertidos, que rebasan la cuarta parte del total nacional en la mayor parte de los casos y en otros superan la sexta parte, con la sola excepción de los beneficios de empresarios y trabajadores autónomos agrícolas, que quedan en la décima parte.

La renta interior, a precios corrientes, que comprende la retribución a los factores de producción - rentas de los factores-y las transferencias, se elevó en el año 1969 a 362.090 millones de pese- 
tas de dicho año, de la que se deduce una renta "per capita» interior de 73.590 pesetas, bastante superior a la nacional, cifrada en 54.760 pesetas.

En orden al consumo se dispone de algunos indicadores muy significativos, que dan idea del nivel de bienestar de la Región. En primer término, según la encuesta de Presupuestos familiares realizada por el Instituto Nacional de Estadística en los años 1964-1965, la estructura del consumo de los hogares catalanes era como sigue:

\begin{tabular}{|c|c|}
\hline & Porcentajes \\
\hline $\begin{array}{ccccccccc} & \ldots & \ldots & \ldots & \ldots & \ldots & \ldots & \ldots & \ldots\end{array}$ & 48,8 \\
\hline 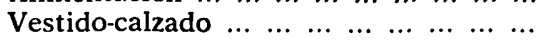 & 15,1 \\
\hline $\begin{array}{lllllllllll}\text { Vivienda } & \ldots & \ldots & \ldots & \ldots & \ldots & \ldots & \ldots & \ldots & \ldots & \ldots\end{array}$ & 7,5 \\
\hline 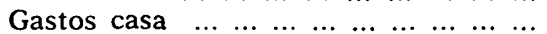 & 8,8 \\
\hline $\begin{array}{lllllllllll}\text { Otros gastos } & \ldots & \ldots & \ldots & \ldots & \ldots & \ldots & \ldots & \ldots & \ldots\end{array}$ & 19,2 \\
\hline $\begin{array}{llllllllll}\text { Vacaciones } & \ldots & \ldots & \ldots & \ldots & \ldots & \ldots & \ldots & \ldots & \ldots\end{array}$ & 0,6 \\
\hline
\end{tabular}

En líneas generales, muy similar a la distribución nacional, pero al detallarse el consumo alimenticio por artículos es de señalar que en la Región catalana se come menos pan, pastas y cereales, patatas y hortalizas, huevos, leche, aceite y azúcar que en el conjunto nacional, mientras se consumen más frutas, carne y pescado, café y bebidas aromáticas, vinos, cervezas y licores.

Según la encuesta de Equipamiento y nivel cultural de la familia realizada en el año 1968 por el Instituto Nacional de Estadística, el equipamiento del hogar catalán en comparación con el del conjunto del país era éste:

\begin{tabular}{|c|c|c|}
\hline & \multicolumn{2}{|c|}{ PORCENTAJES } \\
\hline & Cataluña & $\begin{array}{l}\text { Conjunto } \\
\text { de España }\end{array}$ \\
\hline 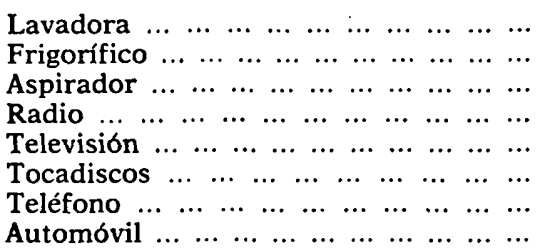 & $\begin{array}{r}57 \\
60 \\
5 \\
86 \\
58 \\
13 \\
31 \\
20\end{array}$ & $\begin{array}{r}40 \\
36 \\
5 \\
76 \\
38 \\
10 \\
19 \\
13\end{array}$ \\
\hline
\end{tabular}

Es evidente que el equipamiento del hogar catalán en su conjunto era en la época de la encuesta superior al del conjunto de España; el grado de equipamiento en la Provincia de Barcelona era 
aún superior, pero difería en las demás Provincias. En Gerona eran menores las disponibilidades de lavadora y de teléfono y aspiradora; en Lérida aparecían menores las disponibilidades de aspiradora y tocadiscos, al igual que en Tarragona, que también tenía inferioridad en cuanto a teléfono se refiere.

En Cataluña se matricularon 119.095 vehículos a motor en 1971, el 2,8 por 100 de los matriculados en toda España. El parque de vehículos en toda Cataluña era de 200 vehículos por cada $1.000 \mathrm{ha}$ bitantes, mientras que la tasa nacional era de 142, nivel superado por todas las Provincias catalanas.

El número de teléfonos por 1.000 habitantes era en 1971 de 221, frente a los 151 del conjunto de España, nivel superado por las Provincias de Barcelona y Gerona.

Finalmente, en cuanto al consumo de productos energéticos, $\mathrm{Ca}$ taluña representa el 14,5 por 100 del total nacional. Su consumo, equivalente a 2.195 TEC por 1.000 habitantes, es inferior al nacional, cifrado en 2.218; sin embargo, éste es superado aisladamente por las Provincias de Gerona y Tarragona.

Hemos tratado de dar una idea de Cataluña en orden a su demografía, su actividad económica, su renta y su consumo y énmarcarla en el conjunto nacional. Ha quedado patente la importancia que en este orden tiene la Región estudiada y el peso que representa en el conjunto nacional.

No hemos utilizado toda la información publicada en la monografía que ha sido objeto de comentario. La vivienda, la enseñanza, la sanidad, la Administración local, el empleo, el comercio exterior y el equipamiento informático no han sido abordados en este trabajo, pero ahí quedan las cifras correspondientes a disposición del estudioso. 
REVL-1974, núm. 182. BALLESTER ROS, IGNACIO. NOTAS SOBRE LA DEMOGRAFIA Y LA ECON... REVL-1974, núm. 182. BALLESTER ROS, IGNACIO. NOTAS SOBRE LA DEMOGRAFIA Y LA ECON... 


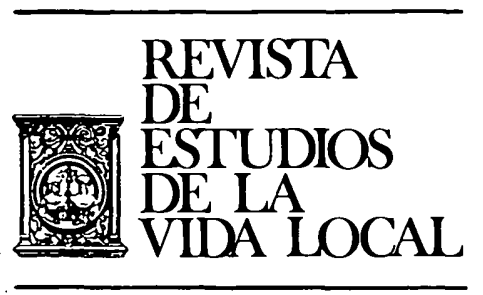

\section{JURISPRUDENCIA}


REVL-1974, núm. 182. BALLESTER ROS, IGNACIO. NOTAS SOBRE LA DEMOGRAFIA Y LA ECON... REVL-1974, núm. 182. BALLESTER ROS, IGNACIO. NOTAS SOBRE LA DEMOGRAFIA Y LA ECON... 